\title{
Effects of a linear central potential induced by the Lorentz symmetry violation on the Klein-Gordon oscillator
}

\author{
R. L. L. Vitória ${ }^{\mathrm{a}}$, H. Belich ${ }^{\mathrm{b}}$ \\ Departamento de Física e Química, Universidade Federal do Espírito Santo, Av. Fernando Ferrari, 514, Goiabeiras, Vitória, ES 29060-900, Brazil
}

Received: 22 October 2018 / Accepted: 23 November 2018 / Published online: 10 December 2018

(C) The Author(s) 2018

\begin{abstract}
Inspired by the Standard Model Extension, we have investigated a possible scenario arising from the Lorentz symmetry violation governed by a background tensor field on a scalar field subject to the Klein-Gordon oscillator, where this possible scenario gives rise to a linear central potential. We analyse the behaviour of the relativistic quantum oscillator under the influence of a Coulomb-type scalar potential in this background. Then, we solve the Klein-Gordon equation analytically and discuss the influence of the background which violates the Lorentz symmetry in the relativistic energy levels.
\end{abstract}

\section{Introduction}

In recent years, in order to observe more accurately the atomic nuclei, some investigations, taken into account the hydrogen atom, have considered to change the orbital electron by a muon, and they have shown that the proton radius is little different [1]. On the other hand, there is a line of research which argue that the fine structure constant $\left(\alpha=\frac{e^{2}}{\hbar c}\right)$ is slowly changing [2,3], and this fact can imply the light velocity variation. Such variation can be consequence of the Lorentz symmetry violation (LSV). It has been extensively studied and applied in various branches of physics [4-15].

The development of the spontaneous symmetry breaking of Lorentz (LSV) in quantum field theory has allowed the formulation of a new model that aims to go beyond the Standard Model which has been known in the literature as Standard Model Extension (SME) $[16,17]$. In the SME there are the fermionic and gauge sectors which are modified by background tensors that are manifested by expected vacuum of more fundamental fields [17]. In particular, the gauge sector is divided in the sectors CPT-odd and CPT-even [18].

\footnotetext{
a e-mails: ricardo.vitoria@pq.cnpq.br; ricardo-luis91@hotmail.com

b e-mail: belichjr@gmail.com
}

In the context of non-relativistic quantum mechanics, through the nonminimal coupling of the term CPT-odd, inspired by gauge sector of the SME, there are studies in geometric phases [18], holonomies [19], on a Dirac neutral particle inside a two-dimensional quantum ring [20], on Aharonov-Bohm-type effect [21] and Landau-type quantization [22]. Recently, also by nonminimal coupling, the CPTeven term, inspired by gauge sector of the SME, has been applied in the analysis of the relativistic quantum dynamics of a scalar field under effects of central potentials induced by the LSV, for example, Coulomb-type potential [23], harmonictype and linear-type potentials [24,25] and the Klein-Gordon oscillator under effects of a Coulomb-type potential induced by the LSV [26]. However, the Klein-Gordon oscillator was not investigated under the effects of a central linear potential induced by the LSV caused by the presence of a background tensor field.

Inspired by the relativistic oscillator model for the spin$\frac{1}{2}$ fermionic field known as the Dirac oscillator [27,28], Bruce and Minning have proposed a relativistic oscillator model for the scalar field which it was known in the literature as the Klein-Gordon oscillator [29] that, in the nonrelativistic limit, is reduced to the oscillator described by the Schoröndinger equation [30]. The Klein-Gordon oscillator has been studied by a $\mathcal{P} \mathcal{T}$-symmetric Hamiltonian [31], in noncommutative space $[32,33]$, in spacetime with cosmic string [34], in a spacetime with torsion [35], in a KaluzaKlein theory [36], with noninertial effects [37] and under effects of linear and Coulomb-type central potentials [3840].

In this paper, we have investigated the effects of a linear central potential induced by the LSV provided by the presence of a background tensor field on a scalar field subject to the Klein-Gordon oscillator. This analysis is made through the nonminimum coupling into the Klein-Gordon equation. Then, we search for relativistic bound state solutions to the 
Klein-Gordon equation, where we have shown that analytical solutions can be achieved.

The structure of this paper is as follows: in Sect. 2, we introduce a possible scenario of the LSV defined by the presence of a background tensor field $\left(K_{F}\right)_{\mu \nu \alpha \beta}$ which governs the LSV out of the SME, and thus, the possible scenario of the LSV induces a linear central potential; in Sect. 3, we generalize our analysis by inserting a Coulomb-type central potential by modifying the mass term of the Klein-Gordon equation and determining solutions of bound states for the scalar field subject to the effects of the LSV; in Sect. 4, we present our conclusions.

\section{Klein-Gordon oscillator under effects of a linear central potential induced by the LSV}

Inspired by the CPT-even sector of the SME [16-18], we can investigate the relativistic quantum dynamic by a massive scalar field under the effects of the LSV by introducing a nonminimal coupling into the Klein-Gordon equation given by $\hat{p}_{\mu} \hat{p}^{\mu} \rightarrow \hat{p}_{\mu} \hat{p}^{\mu}-\frac{g}{4}\left(K_{F}\right)_{\mu \nu \alpha \beta} F^{\mu \nu} F^{\alpha \beta}$, where $g$ is a coupling constant, $F_{\mu \nu}=\partial_{\mu} A_{\nu}-\partial_{\nu} A_{\mu}$ is the electromagnetic tensor and $\left(K_{F}\right)_{\mu \nu \alpha \beta}$ is the constant background tensor field that governs the LSV out of the SME $[16,17]$ and describes the anisotropy of the spacetime by creating preferred directions in the spacetime. Thus, the Klein-Gordon equation is given in the form $(\hbar=c=1)$ :

$\hat{p}_{\mu} \hat{p}^{\mu} \phi-\frac{g}{4}\left(K_{F}\right)_{\mu \nu \alpha \beta} F^{\mu \nu} F^{\alpha \beta} \phi-m^{2} \phi=0$,

where $\hat{p}_{\mu}=i \partial_{\mu}$ and $m$ is the rest mass of the scalar field.

The Klein-Gordon oscillator [29] allows us to write the Klein-Gordon equation in $(3+1)$ dimensions as follows [38]

$$
\begin{gathered}
-\frac{\partial^{2} \phi}{\partial t^{2}}-(\hat{p}+i m \omega \rho \hat{\rho}) .(\hat{p}-i m \omega \rho \hat{\rho}) \phi \\
-\frac{g}{4}\left(K_{F}\right)_{\mu \nu \alpha \beta} F^{\mu \nu} F^{\alpha \beta} \phi-m^{2} \phi=0,
\end{gathered}
$$

where $\rho=\sqrt{x^{2}+y^{2}}$ is the radial coordinate, $\hat{\rho}$ is a unit vector in the radial direction and $\omega$ is the angular frequency of the Klein-Gordon oscillator. Note that we have written the Eq. (2) by using the signature of the spacetime:

$d s^{2}=-d t^{2}+d \rho^{2}+\rho^{2} d \varphi^{2}+d z^{2}$.

Another property of the background tensor field $\left(K_{F}\right)_{\mu \nu \alpha \beta}$ is that it can be written in terms of $3 \times 3$ matrices, $\kappa_{D E}, \kappa_{D B}$, $\kappa_{H E}$ and $\kappa_{H B}$, defined in Refs. [41-43] as:

$$
\begin{aligned}
\left(\kappa_{D E}\right)_{i j} & =-2\left(K_{F}\right)_{0 i 0 j} ; \\
\left(\kappa_{H B}\right)_{j k} & =\frac{1}{2} \varepsilon_{j p q} \varepsilon_{k l m}\left(K_{F}\right)^{p q l m} ; \\
\left(\kappa_{D B}\right)_{j k} & =-\left(\kappa_{H E}\right)_{k j}=\varepsilon_{k p q}\left(K_{F}\right)^{0 j p q} .
\end{aligned}
$$

Note that the matrices $\left(\kappa_{D E}\right)_{i j}$ and $\left(\kappa_{H B}\right)_{j k}$ are symmetric while the matrices $\left(\kappa_{D B}\right)_{j k}$ and $\left(\kappa_{H E}\right)_{k j}$ are antisymmetric. In this way, we can rewrite the Eq. (2) in the form

$$
\begin{aligned}
& -\frac{\partial^{2} \phi}{\partial t^{2}}-(\hat{p}+i m \omega \rho \hat{\rho}) \cdot(\hat{p}-i m \omega \rho \hat{\rho}) \phi-\frac{g}{2}\left(\kappa_{D E}\right)_{i j} E^{i} E^{j} \phi \\
& \quad+\frac{g}{2}\left(\kappa_{H B}\right)_{i j} B^{i} B^{j} \phi \\
& -g\left(\kappa_{D B}\right)_{i j} E^{i} B^{j} \phi-m^{2} \phi=0 .
\end{aligned}
$$

Let us consider a possible scenario determined by the LSV considering the only nonzero component of the constant background tensor field $\left(\kappa_{D B}\right)_{i j}$ being $\left(\kappa_{D B}\right)_{13}=a$ and by a field configuration given by [24]

$\vec{E}=\frac{\lambda}{2} \rho \hat{\rho} ; \quad \vec{B}=B_{0} \hat{z}$,

where $\lambda$ is a constant associated with a volumetric distribution of electric charges, $B_{0}$ is a constant and $\hat{z}$ is a unit vector in the $z$-direction. It is important to note that the electric and magnetic fields given in the Eq. (6) are external independent background fields. This configuration has been studied in induced electric dipole moment systems [44-47] and in LSV possible scenarios [18,24,48,49].

Let us consider a particular solution to the Eq. (5) given in terms of the eigenvalues of the $z$-component of the angular momentum $\hat{L}_{z}=-i \partial_{\varphi}$ and of the eigenvalues of the $z$ component of the linear momentum operator $\hat{p}_{z}=-i \partial_{z}$ as

$\phi(\rho, \varphi, z, t)=e^{-i(\mathcal{E} t-l \varphi-k z)} f(\rho)$,

where $f(\rho)$ is the radial wave function, $l=0, \pm 1, \pm 2, \ldots$ are eigenvalues of the angular momentum and $-\infty<k<$ $\infty$ are eigenvalues of the $z$-component of the linear momentum. Then, substituting the Eqs. (6) and (7) into the Eq. (5), we obtain the ordinary differential equation

$$
\begin{aligned}
& \frac{d^{2} f}{d \rho^{2}}+\frac{1}{\rho} \frac{d f}{d \rho}-\frac{l^{2}}{\rho^{2}} f-\frac{a B_{0} g \lambda \rho}{2} f-m^{2} \omega^{2} \rho^{2} f \\
& +\left(\mathcal{E}^{2}-m^{2}-k^{2}+m \omega\right) f=0 .
\end{aligned}
$$

We proceed with a change of variables given by $\varrho=$ $\sqrt{m \omega} \rho$, and thus, we rewrite the Eq. (8) in the form

$\frac{d^{2} f}{d \varrho^{2}}+\frac{1}{\varrho} \frac{d f}{d \varrho}-\frac{l^{2}}{\varrho^{2}} f-\alpha \varrho f-\varrho^{2} f+\beta f=0$,

where we define

$\alpha=\frac{a B_{0} g \lambda}{2(m \omega)^{3 / 2}} ; \quad \beta=\frac{1}{m \omega}\left(\mathcal{E}^{2}-m^{2}-k^{2}+m \omega\right)$.

By imposing that $f(\rho) \rightarrow 0$ when $\varrho \rightarrow 0$ and $\varrho \rightarrow \infty$, then, the function $f(\varrho)$ can be written as follows:

$f(\varrho)=\varrho^{|l|} e^{-\frac{1}{2} \varrho(\varrho+\alpha)} h(\varrho)$,

where $h(\varrho)$ is an unknown function. 
By substituting the Eq. (11) into the Eq. (9), we note that the function $h(\varrho)$ is a solution to the following second order differential equation:

$$
\begin{aligned}
& \frac{d^{2} h}{d \varrho^{2}}+\left[\frac{(2|l|+1)}{\varrho}-\alpha-2 \varrho\right] \frac{d h}{d \varrho} \\
& +\left[\beta+\frac{\alpha^{2}}{4}-2-2|l|-\frac{\alpha}{2 \varrho}(2|l|+1)\right] h=0 .
\end{aligned}
$$

The Eq. (12) is called as the biconfluent Heun equation [50, 51] and the function $h(\varrho)$ is the biconfluent Heun function, that is, $h(\varrho)=h_{B}\left(2|l|, \alpha, \beta+\frac{\alpha^{2}}{4}-2-2|l|, 0 ; \varrho\right)$.

The Eq. (12) contains two singular points: the origin, a regular singular point, and infinite, an irregular singular point [50]. Since the origin is a regular singular point, the Eq. (12) has at least one solution around this point given by the power series [52]

$h(\varrho)=\sum_{j=0}^{\infty} b_{j} \varrho^{j}$.

Then, from the Eq. (12), we obtain the recurrence relation

$b_{j+2}=\frac{[\delta+\alpha(j+1)] b_{j+1}-(\gamma-2 j) b_{j}}{(j+2)(j+2+2|l|)}$

with the coefficients $b_{1}$ and $b_{2}$ are (where we are considering $\left.b_{0}=1\right)$

$$
\begin{aligned}
b_{1}= & \frac{\delta}{(1+2|l|)}=\frac{\alpha}{2} ; \\
b_{2}= & \frac{1}{2(2+2|l|)}\left[(\delta+\alpha) b_{1}-\gamma\right] \\
& =\frac{1}{4(1+|l|)}\left(\frac{\alpha \delta}{2}+\frac{\alpha^{2}}{2}-\gamma\right),
\end{aligned}
$$

where we define

$\gamma=\beta+\frac{\alpha^{2}}{4}-2-2|l| ; \quad \delta=\frac{\alpha}{2}(2|l|+1)$.

Solutions of bound states can be determined by imposing that the recurrence relation (14) is truncated giving, then, the following conditions

$b_{n+1}=0 ; \quad \gamma=2 n$,

where $n=1,2,3, \ldots$ is the radial mode. With the aim to obtain the energy for the stationary states, let us discuss a particular case of the radial mode $n=1$ which, from the physical point of view, represents the lowest energy state of the system. This means that we want to construct a first degree polynomial to biconfluent Heun series (13). Then, for $n=1$ in the condition $b_{n+1}=0$, we have $b_{2}=0$, then, let us assume that the angular frequency of the Klein-Gordon oscillator can be adjusted in such a way that the condition $b_{n+1}=0$ is satisfied for any value of $n$, and thus, we have obtained the relation

$\omega_{l, 1}=\frac{1}{2 m} \sqrt[3]{\left(\frac{a B_{0} g \lambda}{2}\right)^{2}(2|l|+3)}$.

The Eq. (18) gives us the allowed values for the angular frequency of the Klein-Gordon oscillator corresponding to the radial mode $n=1$. For the lowest energy state of the system, this permit us to construct a first degree polynomial to the biconfluent Heun series (13). Since the allowed values of the Klein-Gordon oscillator frequency are determined by the quantum numbers $\{l, n\}$, we have labelled $\omega=\omega_{l, n}$. In addition, we have that the allowed values of the relativistic quantum oscillator angular frequency depend on the parameters that establish the scenario of the LSV, $a, B_{0}, g$ and $\lambda$.

From the condition $\gamma=2 n$, we obtain the expression

$$
\begin{aligned}
& \mathcal{E}_{k, l, n} \\
& = \pm \sqrt{m^{2}+k^{2}+2 m \omega_{l, n}\left(\frac{1}{2}+n+|l|\right)-\frac{\left(a B_{0} g \lambda\right)^{2}}{16 m^{2} \omega_{l, n}^{2}}},
\end{aligned}
$$

which considering $n=1$ gives us the allowed values of the relativistic energy for the lowest energy state of the quantum system

$$
\begin{aligned}
& \mathcal{E}_{k, l, 1} \\
& = \pm \sqrt{m^{2}+k^{2}+\sqrt[3]{\left(\frac{a B_{0} g \lambda}{2(2|l|+3)}\right)^{2}}\left[\left(\frac{3}{2}+|l|\right)(2|l|+3)-1\right]} .
\end{aligned}
$$

We can note that the presence of the linear central potential induced by LSV modifies the energy spectrum of a scalar field subject to the Klein-Gordon oscillator [29,30]. On the other hand, the lower energy state is determined by the radial mode $n=1$, in contrast to the quantum number $n=0$ as obtained in Ref. [29]. Another quantum effect from the possible scenario of LSV is that the angular frequency of the Klein-Gordon oscillator has restricted values determined by the quantum numbers of the system and the parameters associated with spacetime anisotropy, which allow us to obtain a polynomial solution to the biconfluent Heun series.

\section{Klein-Gordon oscillator under effects of a linear central potential induced by the violation of the Lorentz symmetry and Coulomb-type interaction}

The standard procedure of inserting central potentials into relativistic wave equations is through the minimum coupling $\hat{p}_{\mu} \rightarrow \hat{p}_{\mu}-q A_{\mu}$, with $q A_{0}=V(\vec{r})$, where $q$ is the charge of the scalar field and $V(\vec{r})=V(r)$ is a central potential [53]. Another procedure of inserting central potentials into 
relativistic wave equations is presented in Ref. [53], which is done by modification of the mass term $m \rightarrow m+S(\vec{r})$, where $m$ is the rest mass and $S(\vec{r})$ is the non-electromagnetic central potential. This procedure was used to study Landau quantization in cosmic string spacetime [50], quark-antiquark interaction [54], scalar field in spacetime with torsion [55], the Aharonov-Bohm effect in spacetime with global monopole [56], effects of a central linear potential on a scalar field in Gödel-type spacetime [57] and for a Dirac particle [58]. In this work, we consider a scalar potential proportional to the inverse of the radial distance, then, the mass term of the Klein-Gordon equation becomes $[38,53]$

$m \rightarrow m+\frac{c}{\rho}$,

where $c$ is a constant that characterizes the Coulombtype potential. In the context of quantum mechanics, the Coulomb-type potential was studied in an atom with permanent electric dipole moment $[59,60]$, in an AharonovCasher system [61], in a Kaluza-Klein theory [62] and in electric quadrupole moment systems $[63,64]$. Therefore, by considering the background of the LSV in the previous section, the general form of the Klein-Gordon radial equation which describes the interaction of a scalar field subject to the Klein-Gordon oscillator plus Coulomb-type scalar potential (2) is given by

$\frac{d^{2} f}{d \varrho^{2}}+\frac{1}{\varrho} \frac{d f}{d \varrho}-\frac{\iota^{2}}{\varrho^{2}} f-\frac{\eta}{\varrho} f-\alpha \varrho f-\varrho^{2} f+\beta f=0$,

where we follow the same steps of the Eq. (2) to the Eq. (9) and define the new parameters

$\iota^{2}=l^{2}+c^{2} ; \quad \eta=2 c \sqrt{\frac{m}{\omega}}$.

By analysing the asymptotic behaviour of the possible solutions to the Eq. (23), we can write the function $f(\varrho)$ in terms of an unknown function $H(\varrho)$ as

$f(\varrho)=\varrho^{|\iota|} e^{-\frac{1}{2} \varrho(\varrho+\alpha)} H(\varrho)$.

Then, substituting the Eq. (24) into the Eq. (22), we obtain the biconfluent Heun equation [50,51]:

$$
\begin{aligned}
& \frac{d^{2} H}{d \varrho^{2}}+\left[\frac{(2|\iota|+1)}{\varrho}-\alpha-2 \varrho\right] \frac{d H}{d \varrho} \\
& +\left[\beta+\frac{\alpha^{2}}{4}-2-2|\iota|-\frac{\alpha}{2 \varrho}(2|\iota|+1)-\frac{\eta}{\varrho}\right] H=0,
\end{aligned}
$$

where $H(\varrho)$ is the biconfluent Heun function determined by $H(\varrho)=H_{B}(\varrho)=\left(2|\iota|, \alpha, \beta+\frac{\alpha^{2}}{4}-2-2|\iota|, 2 \eta ; \varrho\right)$. Вy following the steps from the Eq. (13) to the Eq. (15), we obtain the recurrence relation: $b_{j+2}=\frac{[\bar{\delta}+\alpha(j+1)] b_{j+1}-(\bar{\gamma}-2 j) b_{j}}{(j+2)(j+2+2|\iota|)}$,

and the coefficients (with $b_{0}=1$ )

$$
\begin{aligned}
b_{1}= & \frac{\alpha}{2}+\frac{\eta}{(1+2|\iota|)} ; \\
b_{2}= & \frac{1}{4(1+|\iota|)} \\
& \times\left[\frac{\alpha \bar{\delta}}{2}+\frac{\eta \bar{\delta}}{(1+2|\iota|)}+\frac{\alpha^{2}}{2}+\frac{\alpha \eta}{(1+2|\iota|)}-\bar{\gamma}\right],
\end{aligned}
$$

where we define

$\bar{\gamma}=\beta+\frac{\alpha^{2}}{4}-2-2|\iota| ; \quad \bar{\delta}=\frac{\alpha}{2}(2|\iota|+1)+\eta$.

As seen in the previous section, we can determine solutions of bound states by truncating the recurrence relation (26) which gives us the conditions

$b_{n+1}=0 ; \quad \bar{\gamma}=2 n$,

where $n=1,2,3, \ldots$ For $n=1$, as in the previous section, the condition $b_{n+1}=0$ yields $b_{2}=0$. By using the Eq. (27), then, the condition $b_{2}=0$ yields a third degree algebraic equation given by

$$
\begin{gathered}
\omega_{l, 1}^{3}-\frac{2 c^{2} m}{(1+2|\iota|)} \omega_{l, 1}^{2}-\frac{a B_{0} \operatorname{cg} \lambda(1+|\iota|)}{m(1+2|\iota|)} \omega_{l, 1} \\
-\frac{\left(a B_{0} g \lambda\right)^{2}(2|\iota|+3)}{32 m^{3}}=0,
\end{gathered}
$$

where we have labelled $\omega=\omega_{l, n}$. It is well-known that a third-degree algebraic equation has at least one real solution, by the Eq. (30), is the solution that gives the possible values of the angular frequency of the Klein-Gordon oscillator. This effect yields a polynomial solution to the biconfluent Heun series. In particular, the real solution of the Eq. (30) is very long, therefore, we do not write it. In addition, we can also see, from the Eq. (30), that the allowed values of the angular frequency of the Klein-Gordon oscillator depend on the quantum numbers of the system $\{l, n\}$, with parameter associated to the Coulomb-type central potential and the parameters associated with the background of the LSV. Note that, by making $g \rightarrow 0$ we recover the Minkowski spacetime and the allowed values of the angular frequency of the Klein-Gordon oscillator for $n=1$ calculated in Ref. [38]. Now, by making $c=0$, we recover the allowed values of the angular frequency of the Klein-Gordon oscillator calculated in previous section.

From the condition $\bar{\gamma}=2 n$, we obtain the expression

$$
\begin{aligned}
\mathcal{E}_{k, l, n}^{2}= & m^{2}+k^{2}+2 m \omega_{l, n}\left(\frac{1}{2}+n+\sqrt{l^{2}+c^{2}}\right) \\
& -\frac{\left(a B_{0} g \lambda\right)^{2}}{16 m^{2} \omega_{l, n}^{2}}
\end{aligned}
$$


Then, with the real solution of the Eqs. (30) and (31) we can determine the allowed values of the relativistic energy for the radial mode $n=1$, that is, $\mathcal{E}_{k, l, 1}$.

\section{Conclusion}

We have analyzed the effects of LSV on a scalar field subject to the Klein-Gordon oscillator. The background is characterized by the presence of a constant tensor field from LSV, and it has allowed us a possible scenario which induces a linear central potential. The presence of this linear central potential induced by LSV modifies the energy profile of the system and the lower energy state is determined by the radial mode $n=1$ instead of the quantum number $n=0$. By restricting the angular frequency values of the Klein-Gordon oscillator, then the angular frequency of the relativistic oscillator has allowed values defined by the quantum numbers of the system and parameters associated with LSV. Then, a polynomial solution to the Heun biconfluent series can be achieved.

In addition, in order to generalize our analysis by modifying the mass term of the Klein-Gordon equation, we insert a Coulomb-type potential where we have shown that the quantum effects from the influence of the linear central potential and a Coulomb-type potential modify the energy profile of the relativistic oscillator. This restrict the values of the angular frequency of the Klein-Gordon oscillator. They are defined by the quantum numbers of the system and parameters associated with LSV in order to obtain a polynomial solution to the Heun biconfluent series. In this case, we have shown that the allowed values of the angular frequency of the relativistic quantum oscillator associated with the radial mode $n=1$ are determined by a third degree algebraic equation.

Acknowledgements The authors would like to thank the Brazilian agencie CNPq for financial support. R. L. L. Vitória was supported by the CNPq Project No. 150538/2018-9.

Open Access This article is distributed under the terms of the Creative Commons Attribution 4.0 International License (http://creativecomm ons.org/licenses/by/4.0/), which permits unrestricted use, distribution, and reproduction in any medium, provided you give appropriate credit to the original author(s) and the source, provide a link to the Creative Commons license, and indicate if changes were made. Funded by SCOAP ${ }^{3}$.

\section{References}

1. R. Pohl et al., Science 353, 669 (2016)

2. A. Songaila, L.L. Cowie, Nature 398, 667 (1999)

3. A. Songaila, L.L. Cowie, Nature 428, 132 (2004)

4. V.A. Kostelecký, S. Samuel, Phys. Rev. D 39, 683 (1989)

5. H. Belich et al., Rev. Bras. Ensino Fís. 29, 1 (2007)

6. H. Belich et al., Phys. Rev. D 74, 065009 (2006)
7. H. Belich et al., Eur. Phys. J. C 62, 425 (2009)

8. R. Casana et al., Phys. Lett. B 726, 815 (2013)

9. R. Casana et al., Eur. Phys. J. C 74, 3064 (2014)

10. R. Casana, M.M. Ferreira Jr., F.E.P. dos Santos, Phys. Rev. D 90, 105025 (2014)

11. R. Casana, C.F. Farias, M.M. Ferreira, Phys. Rev. D 92, 125024 (2015)

12. R. Casana et al., Phys. Lett. B 746, 171 (2015)

13. G. Gazzola, J. Phys. G Nucl. Part. Phys. 39, 035002 (2012)

14. M. Gomes et al., Phys. Rev. D 81, 045018 (2010)

15. M.B. Cruz, E.R. Bezerra de Mello, AYu. Petrov, Phys. Rev. D 96, 045019 (2017)

16. D. Colladay, V.A. Kostelecký, Phys. Rev. D 55, 6760 (1997)

17. D. Colladay, V.A. Kostelecký, Phys. Rev. D 58, 116002 (1998)

18. K. Bakke, H. Belich, Spontaneous Lorentz Symmetry Violation and Low Energy Scenarios (LAMBERT Academic Publishing, Saarbrücken, 2015)

19. K. Bakke, H. Belich, J. Phys. G Nucl. Part. Phys. 40, 065002 (2013)

20. K. Bakke, H. Belich, Eur. Phys. J. Plus 129, 147 (2014)

21. A.G. de Lima, H. Belich, K. Bakke, Ann. Phys. (Leipzig) 526, 514 (2014)

22. K. Bakke, H. Belich, J. Phys. G Nucl. Part. Phys. 42, 095001 (2015)

23. K. Bakke, H. Belich, Ann. Phys. (NY) 360, 596 (2015)

24. K. Bakke, H. Belich, Ann. Phys. (NY) 373, 115 (2016)

25. R.L.L. Vitória, H. Belich, K. Bakke, Adv. High Energy Phys. 2017, 6893084 (2017)

26. R.L.L. Vitória, H. Belich, K. Bakke, Eur. Phys. J. Plus 132, 25 (2017)

27. M. Moshinsky, A. Szczepaniak, J. Phys. A Math. Gen. 22, L817 (1989)

28. K. Bakke, H.F. Mota, Eur. Phys. J. Plus 133, 409 (2018)

29. S. Bruce, P. Minning, Nuovo Cimento A 106, 711 (1993)

30. N.A. Rao, B.A. Kagali, Phys. Scr. 77, 015003 (2008)

31. J.-Y. Cheng, Int. J. Theor. Phys. 50, 228 (2011)

32. B. Mirza, R. Narimani, S. Zare, Commun. Theor. Phys. 55, 405 (2011)

33. M.-L. Liang, R.-L. Yang, Int. J. Mod. Phys. A 27, 1250047 (2012)

34. A. Boumali, N. Messai, Can. J. Phys. 92, 1 (2014)

35. R.L.L. Vitória, K. Bakke, Int. J. Mod. Phys. D 27, 1850005 (2018)

36. J. Carvalho et al., Eur. Phys. J. C 76, 365 (2016)

37. L.C.N. Santos, C.C. Barros Jr., Eur. Phys. J. C 78, 13 (2018)

38. K. Bakke, C. Furtado, Ann. Phys. (NY) 355, 48 (2015)

39. R.L.L. Vitória, K. Bakke, Eur. Phys. J. Plus 131, 36 (2016)

40. R.L.L. Vitória, C. Furtado, K. Bakke, Ann. Phys. (NY) 370, 128 (2016)

41. A.V. Kostelecký, M. Mewes, Phys. Rev. Lett. 87, 251304 (2001)

42. A.V. Kostelecký, M. Mewes, Phys. Rev. D 66, 056005 (2002)

43. A.V. Kostelecký, M. Mewes, Phys. Rev. Lett. 97, 140401 (2006)

44. Y. Aharonov, A. Casher, Phys. Rev. Lett. 53, 319 (1984)

45. L.R. Ribeiro, C. Furtado, J.R. Nascimento, Phys. Lett. A 348, 135 (2006)

46. C. Furtado, J.R. Nascimento, L.R. Ribeiro, Phys. Lett. A 358, 336 (2006)

47. K. Bakke, C. Furtado, Eur. Phys. J. B 87, 222 (2014)

48. K. Bakke, H. Belich, Ann. Phys. (NY) 354, 1 (2015)

49. K. Bakke, H. Belich, Eur. Phys. J. Plus 127, 102 (2012)

50. E.R. Figueiredo Medeiros, E.R. Bezerra de Mello, Eur. Phys. J. C 72, 2051 (2012)

51. A. Ronveaux, Heuns Differential Equations (Oxford University Press, Oxford, 1995)

52. G.B. Arfken, H.J. Weber, Mathematical Methods for Physicists, 6th edn. (Elsevier Academic Press, New York, 2005)

53. W. Greiner, Relativistic Quantum Mechanics: Wave Equations, 3rd edn. (Springer, Berlin, 2000)

54. M.K. Bahar, F. Yasuk, Adv. High Energy Phys. 2013, 814985 (2013) 
55. R.L.L. Vitória, K. Bakke, Gen. Relativ. Gravity 48, 161 (2016)

56. L. Cavalcanti de Oliveira, E.R. Bezerra de Mello, Class. Quantum Gravity 23, 5249 (2006)

57. R.L.L. Vitória, C. Furtado, K. Bakke, Eur. Phys. J. C 78, 44 (2018)

58. G. Soff et al., Z. Naturforsch. A 28, 1389 (1973)

59. A.B. Oliveira, K. Bakke, Ann. Phys. (NY) 365, 66 (2016)
60. A.B. Oliveira, K. Bakke, Proc. R. Soc. A 472, 20150858 (2016)

61. P.M.T. Barboza, K. Bakke, Eur. Phys. J. Plus 131, 32 (2016)

62. E.V.B. Leite, H. Belich, K. Bakke, Adv. High Energy Phys. 2015, 925846 (2015)

63. K. Bakke, Ann. Phys. (NY) 341, 86 (2014)

64. K. Bakke, Int. J. Mod. Phys. A 29, 1450117 (2014) 